Eduard Palomer, Roser Izquierdo, Eithne Leahy, Carme Masferrer, Paz Flores.

\title{
El usuario como experto: concepto, modalidades y experiencia desde el Proyecto Emilia.
}

\author{
User as an expert: concept, modalities and the \\ experience from the Emilia Project.
}

RESUMEN: Se relata la experiencia del proyecto EMILIA y se ofrece una visión global de qué se entiende por "usuario experto" y "implicación de los usuarios" en salud mental. Se discuten sus implicaciones para los profesionales, usuarios y el sistema sanitario.

PALABRAS CLAVE: Exclusión social, empowerment, recuperación, psicosis, EMILIA.
ABSTRACT: The experience of the EMILIA project is explained and a global vision is offered so as to gain a better understanding about the "Expert by Experience" and user involvement in mental health, the implications for professionals, users and the health system.

KEY WORDS: Social exclusion, empowerment, recovery, psychosis, EMILIA.

La experiencia del proyecto EMILIA en Barcelona.

EMILIA (Empowerment of Mental Illness Service Users: Lifelong Learning, Integration and Action) (1) es un proyecto multicéntrico de investigación que forma parte del sexto programa marco de la Unión Europea. El proyecto se vertebra en tres ejes principales: la formación entendida como formación continuada (LLL), la capacitación de los usuarios (Empowerment) y la legitimación del conocimiento de los usuarios vistos como expertos. En él, participan diferentes instituciones sanitarias y docentes de los siguientes países: Inglaterra, Noruega, Francia, Grecia, España (2), Eslovenia, Polonia, Dinamarca, Bosnia y Suecia.

El proyecto EMILIA pretende mejorar la inclusión social de las personas afectadas de enfermedad mental grave a partir de la hipótesis que la formación y la legitimación del conocimiento que poseen los usuarios, por el mero hecho de serlo, puede incrementar sus posibilidades de encontrar y mantener un trabajo, incrementar el tiempo que están en formación y mejorar, en ultima instancia, su calidad de vida. Además, pretende, para los usuarios que hayan recibido la formación, crear lugares de trabajo dentro de la organización sanitaria como educadores o formadores de otros usuarios con menos experiencia, menos conocimiento sobre la enfermedad y peor manejo psicosocial o experiencia en el uso de servicios.

Para mejorar la capacitación de los usuarios, se desarrollaron entre los países participantes una serie de programas de formación que, de una forma más o 
menos genérica, mejorase las habilidades de un grupo de usuarios afectados de trastornos mentales graves y les introdujera en un nuevo modelo de recuperación (recovery). Los programas estaban estructurados en 10 sesiones cada uno de 1 hora y 45 minutos de duración y eran impartidos por profesionales de Salud Mental (psicólogos, psiquiatras y enfermeras). La metodología de las sesiones seguía el modelos de lifelong learning (LLL), basado en el aprendizaje por la experiencia. El método de participación y diálogo aplicado a la formación pretendía que, en un futuro, los usuarios en formación asumieran el rol de formadores de otros usuarios (peer-to-peer learning) (3). Los módulos impartidos fueron son los siguientes:

Taller Multifamiliar: Trabajo con las familias y los usuarios para mejorar la comunicación, resolver los conflictos de un modo constructivo y dar herramientas para afrontar el estrés.

Red de soporte social: Definición de la red social de la persona, estrategias para adecuarla y para reducir el estigma y la discriminación.

Módulo de recuperación e integración: Basado en el concepto de "empowerment", ayuda a los usuarios a (re)tomar el control de sus vidas: capacitar para tomar todo tipo de decisiones en sus vidas.

Fortalezas: Reconocimiento y aceptación de puntos fuertes y débiles, así como la forma de modificarlos y introspección sobre el propio estigma y sus efectos.

Plan de desarrollo personal (PDP): Ayuda a los usuarios a clarificar sus planes de desarrollo y priorizar opciones vitales. Consiste en relatar su situación pasada, presente y futura en cinco áreas: formación, ocupación, red social, convivencia y ingresos.

Los criterios de inclusión para participar en el proyecto han sido: estar diagnosticado de Esquizofrenia o trastorno Bipolar, ser mayor de edad, tener un mínimo de 3 años de contacto con los servicios de salud mental, no tener trabajo remunerado y haber firmado el consentimiento informado. Para sensibilizar a la organización del papel de la implicación de los usuarios se llevó a cabo un curso de sensibilización sobre el "paciente experto" abierto a todo el personal del hospital. El proyecto, ha capacitado a más de 25 usuarios de salud mental en un programa inespecífico.

La formación realizada en este proyecto ha permitido abrir dos líneas de implicación laboral para los usuarios en el futuro próximo: la primera, la del usuario como docente colaborador y formador en un futuro de otros usuarios y la segunda, la del usuario como mediador entre las instituciones sanitarias y el usuario que es atendido en ellas. En el apartado de las conclusiones comentaremos el desarrollo de estas iniciativas. 
ORIGINALES Y REVISIONES

El usuario como experto:

Exclusión social, recuperación y "Empowerment"

Podemos definir la exclusión social como una combinación de factores interconectados que incluyen bajo nivel de ingresos, precariedad en la vivienda, bajo nivel de habilidades, una pobre educación, importantes dificultades para participar efectivamente de la vida social, política, cultural y económica quedándose así distanciado de la mayoría de sociedad (4). Queda claro que la exclusión social no es, pues, meramente una falta de poder adquisitivo; va más allá de ésta y pone de relieve la pérdida de roles significativos para la persona.

Como ya es sabido, el colectivo de personas que padecen enfermedades mentales graves está entre los más excluidos de la sociedad. La pérdida de estatus social y la discriminación a la que están sometidos no hace más que dificultar su recuperación. Esta situación ha sido denunciada de forma contundente en los últimos años por las asociaciones de usuarios y por usuarios que, aisladamente, han relatado su experiencia de enfermedad y de recuperación (como Patt Deegan (5) o Judi Chamberlin (6)) así como también por profesionales del campo de la rehabilitación psiquiátrica (7). A través y gracias a estas experiencias se ha ido conformando un enfoque orientado a la recuperación que tiene poco que ver con lo que en lenguaje coloquial o en el argot médico entenderíamos por ésta. Así formulada, la recuperación es definida como "un proceso profundamente personal y único de cambio de las propias actitudes, valores, sentimientos, objetivos, habilidades y roles. Se trata de cómo vivir de forma satisfactoria y con esperanza a pesar de las limitaciones causadas por la enfermedad" (8), de desarrollar una identidad que no sea definida por ésta y de reducir su impacto en la vida diaria. Esta experiencia de recuperación tiene, pues, más que ver con la superación de las consecuencias psicosociales y económicas que comporta la enfermedad mental que con la mejora de los síntomas (algo por lo que, a veces, los profesionales estamos excesivamente preocupados y que nos resta capacidad para atender a la persona en todas sus áreas) (6). Recuperarse implica también (re)descubrir un sentido positivo de si mismo y unas relaciones sociales satisfactorias que permitan desempeñar roles significativos. Este proceso es indisociable de otro: la recuperación de la esperanza. Esto es algo que ya sabíamos: que las expectativas que los profesionales tienen sobre el pronóstico y capacidades de sus pacientes se transmiten inevitablemente a éstos últimos (9). Se presume también que este proceso es único y diferente por cada persona. Está claro que todos definiríamos qué significa "vivir bien" de una forma distinta, poniendo el acento en unos aspectos y no en otros. Ésta singularidad en la recuperación no significa que los usuarios no se beneficien de "settings" comunes como son los centros de rehabilitación psicosocial (CRP) o las unidades de salud mental (USM). Todo lo contrario: 
son necesarios pero deben cambiar para adaptarse a un modelo más próximo a lo comunitario que a los modelos institucionales. Un cambio básico es ver a los usuarios y sus capacidades como capital valioso en el que los servicios se pueden apoyar y no verlos sólo como el mero receptor pasivo de los servicios, $(7,10)$. Tal y como nos dice Mary O’Hagan (una usuaria nuevazenlandesa experta en recuperación) "la recuperación sólo tiene lugar cuando las personas tienen un rol activo en la mejora de sus vidas" (11).

El proceso de recuperación comporta necesariamente pues el ganar otra vez el control sobre la propia vida y eso tiene que ver con tomar decisiones (sean estas a veces acertadas o no). Esto es lo que en la literatura se le da el nombre de empowerment. Este concepto, que está actualmente muy de moda (la mayoría de programas afirma que este es uno de sus objetivos), es difícil de traducir con una sola palabra a nuestra lengua. La mayoría de definiciones hace alusión a un proceso por medio del cual los usuarios tienen poder para tomar decisiones, disponen de información necesaria para tomarlas, son capaces de actuar asertivamente, creen en sus posibilidades de cambio y tienen un fuerte sentimiento de autoeficacia,(12)(6). Durante muchos años hemos justificado restringir las libertades de la persona en pro de su beneficio. Todos los que trabajamos con personas afectadas de trastornos mentales graves sabemos que actuar de forma paternalista es siempre una tentación (sea porqué es mas cómodo y menos arriesgado para ellos que decidamos nosotros o porqué creemos que su decisión será errónea). Además, no siempre somos conscientes de ello (escogiendo sutilmente información a favor de lo queremos que hagan/decidan) $(13,14)$. El famoso empowerment reivindica, pues, el derecho a equivocar-se, pero informado.

En diferentes documentos-guía $(7,11)$ se han descrito de forma detallada cuales son las competencias y cualidades que debe tener los profesioanles o los servicios para trabajar hacia la recuperación. A modo de resumen cabe destacar las que se plantean, como fundamentales desde el sistema de salud mental nuevazelandés (11):

- Promueve la autonomía como principio fundamental de la recuperación.

- Sabe "ver" a las personas en su amplio contexto de vida (no sólo a través de la enfermedad).

- Está familiarizado con el concepto de la resiliencia y los principios de la educación de adultos como más significatives.

- Ayuda a los usuarios a que vivan de la forma que han escogido (les ayuda a encontrar vivienda adecuada, en el manejo de las responsabilidades cotidianas, en el establecimiento y mantenimiento de relaciones significativas...)

- Es capaz de acomodar los diferentes enfoques y tratamientos de los trastornos mentales en los diferentes servicios y adaptar esta información para promover la recuperación. 
ORIGINALES Y REVISIONES

- Tiene habilidades para informar a los usuarios sobre las múltiples opciones orientadas hacia la recuperación.

- Sabe detectar y manejar el estigma internalizado.

- Tiene habilidades para articular acciones contra los actos discriminatorios (sea en el ámbito laboral, de salud, familiar, de ocio, legislativo...).

- Conoce y protege activamente los derechos de los usuarios.

- Conoce y promueve los principios de autonomía y autodirección, el derecho a recibir tratamiento y a rechazarlo.

- Conoce las asociaciones/movimientos de usuarios y da soporte a su participación en los servicios.

- Conoce las diferentes formas de participación de los usuarios (por ejemplo, sabe trabajar en colaboración con ellos, escoger a representantes de su perspectiva, "ve" a los usuarios como expertos por la experiencia, da soporte a las iniciativa llevadas a cabo por usuarios de forma independiente...).

- Conoce y da soporte a los familiares y sus perspectivas.

\section{El usuario como experto y su implantación en el campo de la salud mental}

En esta parte vamos a definir qué se entiende como usuario experto en el campo de la salud y, más concretamente, en el ámbito de la salud mental. Analizaremos su relevancia para el sistema sanitario y para los usuarios en si mismos. Finalmente, analizaremos cómo puede convertirse en una realidad a distintos niveles.

El usuario experto. Vivir con una enfermedad crónica genera en la persona que la padece y su entorno una serie de necesidades: reconocer síntomas y actuar ante ellos, manejarse con los fármacos y los servicios, compaginar la vida cotidiana con los cuidados que la enfermedad requiere (15)... En resumen, se debe aprender a manejar la enfermedad en si misma y sus consecuencias físicas, psíquicas y sociales. Por lo tanto, estas necesidades nos obligan a desarrollar habilidades que, de no padecer la enfermedad, no tendríamos. Cuando hablamos de paciente experto nos referimos precisamente a esto: a la convicción que los usuarios (y sus personas más próximas) por el mero hecho de serlo disponen de un conocimiento útil y valioso al que no podemos llegar por otras vías. Así, mientras los clínicos tienen habilidades para: hacer buenos diagnósticos, proponer las opciones terapéuticas adecuadas, valorar el pronostico de los trastornos ,estudiar su origen... Los usuarios las tienen para: conocer las circunstancias sociales en las que se padece la enfermedad, la vivencia subjetiva de ésta, las actitudes de riesgo y los valores y preferencias (16). Estas habilidades, experien- 
cias y conocimientos adquiridos por los usuarios han sido durante mucho tiempo un recurso infrautilizado (17). Podría haber ayudado a mejorar la calidad de vida de los usuarios si no hubiera sido ignorado. La Salud Mental y la investigación tiene una larga historia de ver a los usuarios como un objeto científico creando conocimiento que siempre ha sido desarrollado desde el punto de vista de un observador (18). Mas adelante veremos ejemplos concretos de cómo dar un giro y aprovechar esto para mejorar la vida de los usuarios y, a la vez, la asistencia que reciben en los servicios.

La consecuencia inmediata de asumir esta premisa implica cambiar radicalmente nuestra forma de mirar a los usuarios. Esto significa pasar de una mirada centrada en lo defectuoso, lo que debería estar y no está, lo que sobra...a mirar de qué recursos dispone la persona que podamos utilizar como cimientos de la recuperación. Este cambio tiene, pues, su trascendencia en la praxis diaria. Si no fuera así, nos quedaríamos en mero ejercicio intelectual y voluntarioso, en una bonita declaración de intenciones políticamente correcta (19). La consecuencia directa es convertir a los usuarios en parte implicada en todo el proceso de la toma de decisiones sobre su tratamiento y atención recibida. Sobretodo en los países de habla inglesa existen guías de cómo trabajar en esta dirección no solo en el campo de la salud mental si no también en la mayoría de especialidades médicas. Y esto es, en parte, gracias que gran parte de las personas afectadas de enfermedades crónicas disponen de tratamientos que les permiten vivir más tiempo y mejor. Lo que les permite pasar de ser un mero receptor pasivo de cuidados a, tener mucho qué decir sobre cómo abordar los problemas que padecen.

Un claro ejemplo de trabajar desde estas premisas es el auge de los programas de autogestión en muchas patologías crónicas (20). A pesar de esto, en una reciente revisión de la biblioteca Cochrane se pone de manifiesto que hace falta un mayor control y una selección adecuada de las variables en los futuros estudios (por ej., impacto sobre la propia enfermedad y autoeficacia) y más estudios longitudinales (21).

\section{La Implicación de los usuarios en los servicios}

Como dijimos en el apartado anterior, el primer paso hacia la consideración práctica del usuario como experto es implicarlos en la toma de decisiones que les afectan (22). En este apartado veremos qué se entiende por implicación ("involvement"), sus distintas formas y niveles, las razones para fomentarla y las dificultades que surgen para llevar a cabo políticas al respecto.

Implicar a los usuarios significa algo más que hacerle partícipes de los aspectos relacionados con su tratamiento; intenta ir más allá; pretende que los usua- 
ORIGINALES Y REVISIONES

rios se involucren en la investigación, formación, evaluación y desarrollo de los servicio. Por lo tanto, el concepto de "user involvement" (23-24), (tal y como es conocido en la literatura anglosajona) requiere trabajar con los usuarios como iguales en su propio cuidado y en la adaptación de los servicios a sus necesidades y preferencias. A la práctica esto se traduce en que los usuarios asuman diferentes roles y mayores responsabilidades en todo lo que tiene que ver con los servicios en los que son atendidos. Como veremos al final de este apartado, esto representa inevitablemente un cambio en los juegos de poder en las organizaciones sanitarias. Si no es así, solo se conseguirá quedar bien con los colectivos de usuarios y familiares y no las prácticas implantadas no tendrán efectos positivos en su recuperación ni en la mejora de los servicios.

Existen diferentes formas de implicación de los usuarios. Vamos a diferenciarlas teniendo en cuenta 3 variables (25):

- El nivel de interacción con la organización: Desde la pura interacción entre usuarios hasta la interacción directa con los planificadores pasando por la interacción profesional-usuario y con los proveedores de servicios locales.

- El grado de control/capacidad de influencia sobre ésta: Este va desde la manipulación (como paradigma de la nula capacidad de control) hasta el control social (como metáfora de la máxima capacidad de influencia) pasando por el ser informado, consultado, trabajar juntos o delegar el control. También podemos ver esta variable como un contínuum que va desde el ser un receptor pasivo de cuidados a participar activamente de la forma en que éstos son implementados y planificados.

- El área de influencia potencial: Desde el propio tratamiento hasta la planificación de servicios pasando por la atención directa recibida.

Un primer nivel de participación es aquel en el que la interacción se da entre usuarios. O sea, cuando no hay dialogo con la organización sanitaria. Difícilmente, a este nivel, se tiene capacidad de influencia sobre los servicios. Se refiere, más bien, a las distintas formas de autoayuda con buen impacto en el colectivo de usuarios y familiares pero que difícilmente traspasa a otros ámbitos. A nivel práctico se materializa en la publicación documentos informativos, publicaciones periódicas, grupos de autoayuda... Esto no significa que los colectivos de usuarios no tengan potencialmente influencia en la organización sino que hay el riesgo que sus prácticas sean demasiado endogámicas quedando atrapados en un bucle cerrado en si mismo. Un rol importante a este nivel es la defensa de los y sus derechos, sobretodo de aquellos más vulnerables y con cotas más bajas de "empowerment".

Otro nivel al que la mayoría ya estamos acostumbrados es en la toma de decisiones sobre el tratamiento. Aunque parezca una obviedad, para hacer partícipe a los usuarios en este campo es necesario haberlos informado sobre el plan de tratamiento, acordarlo y dar opciones sobre éste, informar sobre el diagnostico y 
tener un plan sobre como actuar en caso de crisis. A este nivel interactúan ya el usuario y los profesionales pero ambos a nivel individual.

A un nivel cualitativamente diferente está la interacción entre los usuarios y los centros dónde son atendidos. La gama de roles que pueden desarrollar los usuarios aquí es muy amplia y también la trascendencia de su participación es de amplio rango (desde la mera consulta hasta la decisión vinculante). Pueden participar en (26):

- La formación de los profesionales.

- La selección de éstos.

- La dirección de los recursos.

- El trato recibido.

- La evaluación del servicio.

- Las actividades que promueven la recuperación.

Finalmente, los usuarios pueden estar implicados en la planificación de los servicios y en las políticas sanitarias. En este tipo de decisiones es más propio que sean los representantes de los colectivos de usuarios los que actúen como interlocutores. Es importante, pues, que se asegure la representatividad de estos y su sensibilidad hacia las preferencias del colectivo. Así, pueden participar en comités de empresa y equipos de trabajo en los equipos de gobierno.

Hay muchas razones tanto practicas como éticas para involucrar a los usuarios. Unas tienen que ver con los beneficios para la organización y otros con los beneficios que se derivan directamente del proceso de participación por parte de los usuarios. La organización va a beneficiarse del cambio de modelo enriqueciendo y promoviendo el desarrollo de sus profesionales. Estos ganaran feedback de los usuarios, verán una nueva perspectiva de las necesidades y preferencias de los usuarios. Esto les va a permitir reevaluar su propia práctica diaria. En definitiva, esto hará que el sistema sea más sensible a las necesidades de los usuarios. Éticamente, la organización también va a resolver el problema de la desigualdad de poder porque éste sería compartido como también lo sería la responsabilidad. La implicación de los usuarios, en definitiva, va a favorecer la comunicación entre estos y el staff, reduciendo, gracias al contacto, prejuicios mutuos basados en estereotipos muy arraigados.

Para los usuarios la participación es una gran oportunidad para poner en práctica habilidades que han quedado atrofiadas debido, en parte, a la pérdida de oportunidades para practicarlas. Llegamos pues a la conclusión que la participación será terapéutica: mejoraran su autoestima, sentimiento de autoeficacia y de pertenencia al grupo. En definitiva, les ayudará a reducir la exclusión social a través del ejercicio de roles valiosos para ellos, de la co-construcción de una red de apoyo, de dar un sentido a su vida. 
ORIGINALES Y REVISIONES

A pesar de todos estos aspectos, la participación se encuentra con numerosos retos a superar (27). Algunas de las dificultades las causa la propia estructura de los servicios. Otras, de los prejuicios y miedos de los profesionales. Las ultimas vienen, obviamente, de los usuarios y la propia patología. En la Tabla 3 (28) se muestran éstas dificultades ordenadas cruzando dos variables:

- Estructural/Cultural

- Tipo: individual/organizacional/social

Si nos centramos primero en los factores individuales, tenemos que hablar de falta de motivación y del miedo una descompensación que un proceso de este tipo puede favorecer por los niveles de estrés que representa. Otro problema es la necesidad de muchos usuarios de, una vez recuperados, apartarse de todo aquello que tiene que ver con la salud mental. Nos referimos a usuarios que tienen cierta red social fuera de las redes asistenciales, suficientes capacidades para sostenerse sin ellas y cierto grado de estigma internalizado. Para ellos, estar tan lejos de los servicios como sea posible es una señal inequívoca de recuperación. Otros usuarios no quieren participar por un motivo diferente: la desconfianza hacia los servicios. Creen que pueden ser etiquetados como personas excesivamente críticas con el sistema y verse afectada la atención que de él reciben.

La forma en que las organizaciones sanitarias están estructuradas dificulta mucho que los usuarios puedan participar de sus encuentros. En estos se suele utilizar un argot médico quizá incomprensible para los usuarios. Los profesionales a veces tampoco favorecen la participación argumentado que el usuario que está interesado no es quizá representativo de la voz del colectivo. Otro problema que frena la participación es el sistema de beneficios por invalidez: los usuarios no quieren arriesgarse a perder sus pensiones si desde la organización se les ofrece retribución por su participación.

Las actitudes de los profesionales son un factor muy relevante para favorecer o frenar la participación. Estos suelen tener una actitud ambigua: hay muchas diferencias entre el soporte expresado y el que, de hecho, practican. Parece que estas dificultades tienen que ver con la idea de compartir y transferir poder a los usuarios y con dificultades a la hora de verlos como expertos.

\section{Experiencias de participación y su eficacia}

En este aparado pasaremos de la retórica a mostrar a mostrar algunos ejemplos de participación real centrándonos, básicamente, en aquellas llevadas a cabo en los países de habla inglesa. 
La consulta como forma incipiente de participación: Cuando la participación se limita a la consulta a los usuarios sobre sus preferencias es fácil que no estemos delante de una participación real sino delante de un mero acto simbólico con poca trascendencia y capacidad potencial de cambio. La consulta solo será una práctica real de participación si tiene consecuencias en la organización y se mejora, así, el ajuste entre las necesidades de los usuarios y los servicios donde se atienden. Una buena práctica en este sentido es el CUES Project (29). En este proyecto inglés se pretende valorar el impacto de las expectativas de los usuarios y cuidadores per medio de una escala (cubre áreas tales como la vivienda, red social y familia, satisfacción, relación con los profesionales...). El cuestionario permite valorar la situación real y la ideal en cada ítem. El objetivo final de la consulta es mejorar los planes de trabajo permitiendo que los coordinadores del caso conozcan cuáles son los problemas que les afectan. A partir del instrumento de consulta los usuarios podían proponer cambios y mejoras. A pesar de algunos problemas de deseabilidad social éste método se ha mostrado eficaz para corresponsabilizar a los usuarios de la asistencia recibida y ajustarla a sus necesidades y preferencias.

Los programas de automanejo: se trata de aquellos programas llevados a cabo por usuarios que, una vez entrenados, asumen el rol de tutores de otras personas con su misma condición. Se basan en las teoría de Bandura sobre la autoeficacia. Pretenden ayudar a mejorar el manejo de la patología pero también de sus consecuencias en la vida de la persona (reconocer y afrontar síntomas, hacer buen uso de los recursos, afrontar el estigma...). La clave de estos programas es el desarrollo de un sentimiento de competencia percibida, prerrequisito básico para que se dé cualquier cambio de conducta. Dos buenos ejemplos de esto son el Rethink selfmanagement project y el Selfmanagement Training Programme (SMTP) for Manic Depression. Lawn et al. relata, también a través de un programa de automanejo, cambios en las siguientes áreas: Comprensión y manejo de síntomas, aumento de la voluntad para participar de las decisiones sobre su salud, disminución de las hospitalizaciones, cambios en la percepción de los profesionales sobre las habilidades de los usuarios y, de forma global, mejora de la sensación de control.

La implicación como formadores: un ejemplo de lo extendida que está esta práctica es su obligatoriedad desde el año 2005 en el Reino Unido para aquellos formándose como psiquiatras (30). La mayoría de experiencias a este nivel (30-32), advierten del riesgo de convertir la formación en una exhibición de síntomas y, por otra parte, de la necesidad de preparar a los alumnos para encajar bien esta práctica. Además, para facilitar que la experiencia sea exitosa es necesario adaptar el contexto formativo a personas poco acostumbradas a ello. Si todo esto es tenido en cuenta la experiencia demuestra que estas prácticas son útiles para romper ciertos mitos sobre las personas con enfermedad mental y para mejorar las habilidades de los futuros profesionales, por ejemplo, a la hora de llevar a cabo entrevistas clínicas. 
ORIGINALES Y REVISIONES

La implicación como en la planificación y desarrollo de los servicios: Debajo de esta práctica hay la idea de que a través de esta practica se mejoraran los servicios. Serán más accesibles y fomentaran más la calidad de vida de los pacientes. En una revisión sistemática de ésta forma de participación (33) se concluye que falta evidencia (o esta es contradictoria) sobre los efectos que esta practica tiene en sus participantes. Parece que una posible razón de esto seria la no adecuación de los foros de participación a las dificultades de los usuarios o su falta de entrenamiento. Más claros parecen los beneficios para la organización y para romper prejuicios de los profesionales.

La implicación en la investigación: Los usuarios suelen poner el foco de atención en áreas que quizá los profesionales nunca investigaríamos, como prioritarias. Además, la participación en la investigación por parte de usuarios puede ayudar a aumentar su validez ecológica (por ejemplo, participando como entrevistadores de otros usuarios). Un ejemplo de buen praxis al respecto es el trabajo de investigación qualitativa que ADEMM (Usuaris de Salut de Catalunya) publicó sobre la relación entre los usuarios y los profesionales. Trabajo que en estos momentos está en proceso de ampliación.

\section{Conclusiones:}

El proyecto EMILIA, a partir de la interrelación de los conceptos aquí expuestos, ha permitido la creación de puentes entre campos, a veces tan alejados pero tan interdependientes a la vez, como son la salud, el trabajo y la formación.

- El proyecto finalizará a principios del 2010 después de un desarrollo de 5 años.

- Los ocho países que han colaborado como grupo experimental habrán completado la formación de los usuarios de servicios de salud mental y se habrá creado una red europea de investigación europea.

- Los resultados del proyecto tanto cualitativos como cuantitativos no serán publicados hasta su finalización.

- En cuanto a la formación, cabe recordar que los once programas de formación fueros diseñados por el conjunto de colaboradores europeos y cada grupo experimental eligió los programas más adecuados a sus necesidades.

- Respecto a la metodología (LLL), podemos señalar que se han dado cambios en el equipo EMILIA en cuanto a la concepción y realización de la formación y que se ha promocionado la organización de aprendizaje dentro del hospital.

- Ha mejorado la aceptación de los usuarios como participante en algunos 
ámbitos de la organización. Los usuarios ya han iniciado, de manera informal, el trabajo como expertos por la experiencia.

Ha sido a partir de nuestra participación en este proyecto que hemos planteado la posibilidad de continuar la experiencia con dos iniciativas:

En primer lugar, la colaboración de los usuarios de salud mental en la docencia (Universidad o escuelas profesionales) y como formadores de otros usuarios. Los usuarios, formados en docencia a raíz del proyecto, pueden impartir cursos orientados a la recuperación y empowerment, tanto a otros usuarios como a distintos futuros profesionales de salud mental.

En segundo lugar, desarrollar una nueva profesión, basada en la experiencia de enfermedad, que legitimice el conocimiento del experto por la experiencia (EpE). Esta nueva profesión permitirá a los usuarios de servicios de salud mental trabajar como mentores dentro de las instituciones sanitarias.

Para ello hemos diseñado y estamos realizando una formación complementaria. Los nuevos programas han sido desarrollados conjuntamente por el equipo EMILIA, los usuarios, otros colaboradores del hospital Fòrum del Hospital del Mar y docentes de la escuela de formación profesional Bonanova y de la escuela de enfermería de la Universidad (UPF). Estos nuevos programas son acreditados por la Conselleria d'Educació de la Generalitat de Catalunya y subvencionados por el SOC (Servei d'Ocupació de Catalunya).

Como ya se ha dicho anteriormente, esto nos ha permitido establecer vías de colaboración entre la asistencia, la docencia y el mundo laboral. Y creemos que en un futuro nos facilitará ampliar la colaboración laboral de los usuarios y favorecer, a los estudiantes -futuros profesionales de la salud- una nueva perspectiva de la salud mental. También posibilitará romper algunos prejuicios arraigados en la clase médica. Como se deduce, el conocimiento sobre padecer una enfermedad y ser usuario de servicios se legitima como contenido formativo.

También como resultado del proyecto se ha constituido un grupo de usuarios como Asociación EMILIA. Esto nos informa sobre la salud del modelo de trabajo participativo que se ha seguido favoreciendo prácticas de cooperación entre usuarios dónde los profesionales sólo tienen un rol de soporte.

Así pues, el proyecto tiene consecuencias prácticas más allá de su finalización y permite no interrumpir procesos largos como la recuperación. En todo momento, la relación entre usuarios, profesionales de la salud mental y docentes ha favorecido un cambio de vinculación entre ambos.

En resumen, parece que la implicación exitosa de los usuarios en los servicios de salud mental requiere de una preparación considerable tanto por parte de los usuarios como por la organización pero ésta no es imposible y el beneficio potencial para todos es, potencialmente, muy grande. 
Tabla 1

Componentes principales del enfoque orientado a la recuperación. *

\begin{tabular}{|ll|}
\hline $1-$ & Proceso autodirigido \\
\hline $2-$ & Proceso individualizado y centrado en el usuario \\
\hline $3-$ & Potencia el empowerment y el respeto por las decisiones tomadas \\
\hline $4-$ & Puede ser no lineal \\
\hline $5-$ & Se apoya en las capacidades y fortalezas del individuo \\
\hline $6-$ & Potencia la responsabilidad del proceso en el usuario \\
\hline $7-$ & La esperanza como base \\
\hline $8-$ & Emfatiza el soporte que dan los iguales \\
\hline $9-$ & És Holístico y tiene en cuenta al ser humano en todas sus facetas \\
\hline
\end{tabular}

* Adaptado de: Care Services Improvement Partnership (CSIP). A common purpose: Recovery in future mental health services. Great Britain: Social Care Institute for Excellence, 2007.

Tabla 2

Elementos esenciales del concepto de empowerment*

\begin{tabular}{|ll|}
\hline $1-$ & Poder para tomar decisiones y partir de un rango de opciones. \\
\hline 2- & Acceso a la información y recursos. \\
\hline 3- & Capacidad para redefinir-se y redefinir la relación con las instituciones. \\
\hline 4- & Sentimiento de pertenencia al grupo. \\
\hline 5- & Conocimiento de los derechos propios. \\
\hline 6- & Capacidad para cambiar la percepción que los otros tienen de uno mismo y sus capacidades. \\
\hline 7- & Capacidad para fomentar una imagen positiva de si mismo y superar el estigma internalizado. \\
\hline 8- & Capacidad de actuar de forma asertiva y con esperanza. \\
\hline
\end{tabular}

* Adaptado de: Chamberlin J. A working definition of empowerment. Psychiatr Rehabil J 1997;20(4):43-47.

Tabla 3

Dificultades para la participación

\begin{tabular}{|l|l|l|l|}
\hline & \multicolumn{1}{|c|}{ Del individuo } & \multicolumn{1}{c|}{ De la organización } & \multicolumn{1}{c|}{ De la sociedad } \\
\hline Estructural & & $\begin{array}{l}\text { - La naturaleza de los encuentros. } \\
\text { - Representatividad. } \\
\text { - El sistema de pensiones }\end{array}$ & \\
\hline Cultural & - Experiencia de enfermedad. & - Actitudes de los profesionales \\
& - Actitud hacia los servicios. & y usuarios. & \\
\hline
\end{tabular}




\section{BIBLIOGRAFÍA:}

(1) Emilia (Empowerment of Mental Illness Service Users: Lifelong Learning, Integration and Action). [página web en internet]. Disponible en: http://www.emiliaproject.net/.

(2) PAlomer E, IzQuierdo R, Flores P. Per la inclusió social i laboral dels malalts mentals. Presentació del Projecte EMILIA. Revista del COPC 203:19-21.

(3) G Turner, J ShePHerd. A Method In Search of a Theory: Peer Education and Health Promotion. Health Educ Res 1999;12:235-47.

(4) SAYCE L. Social inclusion and mental health. Psychiatr Bull 2001;25:121-3.

(5) DeEgan P.E. Recovery as a Self-Directed Process of Healing and Transformation. Occup Ther Ment Health 2001;17:5-21.

(6) Chamberlin J. A working definition of empowerment Psychiatr Rehabil J 1997;20(4):43-47.

(7) Care Services Improvement Partnership (CSIP). A common purpose: Recovery in future mental health services. Great Britain: Social Care Institute for Excellence, 2007.

(8) Anthony W. A. Expanding the evidence base in an era of recovery [editorial]. Psychiatr Rehabil J 2003;27(1):1-2.

(9) Alexander J.A, Lichtenstein R, Daunno T. A, Mccormick R, Muramatsu N, Ullman E. Determinants of mental health providers' expectations of patients' improvement. Psychiatr Serv 1997;48(5):671-7.

(10) Doughty C. Tse S. The Effectiveness of Service User-Run or Service User-Led Mental Health Services for People with Mental Illness: a systematic literature review. New Zealand: Mental Health Commission Wellington, 2005.

(11) Mental Health Advocacy Coalition. Destination: recovery. Te unga ki uta: te oranga. Auckland (NZ): Mental Health Foundation of New Zealand, 2008.

(12) Rogers E.S, Chamberlin, J, Crean, T. A Consumer-Constructed Scale to Measure Empowerment Among Users of Mental Health Services. Psychiatr Serv 1997;48(8):1042-7.

(13) BreEZE J. Can paternalism be justified in mental health care? J Adv Nurs 1998; 28(2): 260-5.

(14) WoOdward V.M. Caring, patient autonomy and the stigma of paternalism. J Adv Nurs 1998;25(5):1046-52.

(15) Department of Health. The expert patient: A new approach to Chronic Disease Management for the 21st Century. London (UK): 2001.

(16) Coulter A. Engaging Patients In Their Health: How The Nhs Needs To Change. Oxford (UK): Picker Institute, 2007. Disponible en: http://www.pickereurope.org/item/document/204

(17) National Schizophrenia Fellowship. Self-Management: The experiences and views of selfmanagement of people with a diagnosis of schizophrenia. Rethink severe mental illness. UK: 2003. Disponible en: http://www.rethink.org/document.rm?id=109

(18) EX-IN (EXperienced INvolvement). [página web en internet]. Bremen: Initiative zur sozialen Rehabilitation e.V. Disponible en: http://www.ex-in.info.

(19) Hansen T, Hatling T, Lidal E, Ruud T. The user perspective. Respected or rejected in mental health care?. J Psychiatr Ment Health Nurs 2004;11:292-7.

(20) Lawn S, Battersby M.W, Pols R.G, Lawrence J, Parry T, Urukalo M. The mental health expert patient: finding from a pilot study of a generic chronic condition self-management programme for 
ORIGINALES Y REVISIONES

people with mental illness. Int J Soc Psychiatry 2007;53(1):63-74.

(21) Foster G, Taylor S.J.C, Eldridge S.E, Ramsay J, Griffiths C.J. Self-management education programmes by lay leaders for people with chronic conditions. Cochrane Database Syst Rev 2008;4.

(22) KaVAnagh M. An introduction to service user involvement and participation: obstacles and opportunities. Division of Clinical Psychology AGM 2008. Disponible en http://www.bps.org.uk/downloadfile.cfm?file_uuid=A9C2430F-1143-DFD0-7E74-422B9E9104\&ext=ppt

(23) Kamaldeep B, Aubin A, StrathdeE G. Making a reality of user involvement in community mental health services. Psychiatr Bull 1998;22:8-11.

(24) Simpson E.L, HousE A.O. User and carer involvement in mental health services: fron rhetoric to science. Br J Psychiatry 2003;183:89-91.

(25) Svennevig J. An evaluation of the level of service user involvement in the redcar and cleveland locality with an exploration of factors constraining progress and how these might be overcome. Cleveland: UK; 2004. Disponible online en http://www.redcar-cleveland.gov.uk/pdf/SUIRCMHS.pdf

(26) LyNDA T, Lester H. Encouraging user involvement in mental health services. Adv Psychiatr Treat 2005;11:168-75.

(27) Lammers J, Happell B. Consumer participation in mental health services: looking from a consumer perspective. J Psychiatr Ment Health Nurs 2003;10:385-92.

(28) Adaptado de: Svennevig J. An evaluation of the level of service user involvement in the redcar and cleveland locality with an exploration of factors constraining progress and how these might be overcome. Cleveland(UK): 2004. Disponible online en http://www.redcar-cleveland.gov.uk/pdf/SUIRCMHS.pdf

(29) Blenkiron P, Hong Mo K, Cuzen J, Hammill, A.C. Involving service users in their mental health care: the CUES Project. Psychiatr Bull 2003;27:334-8.

(30) ViJayakrishnan A, Rutherford J, Miller S.M Drummond, L.M. Service user involvement in training: the trainees' view. Psychiatr Bull 2005;30:303-5.

(31) CRAWFord M.M, DaviES S. Involvement of users and carers in the training of psychiatrists: making it happen. Psychiatr Bull 1998;2242-43.

(32) Iккоs G. Enganging patients as teachers of clinical interview skills. Psychiatr Bull 2003;27:312-5.

(33) Crawford M.J, Rutter D, Manley C, Weaver T, Bhu K, Fulop N, et al. Systematic review of involving patients in the planning and development of health care. Br Med J 2002;325:1263-65.

* EDUARD PALOMER. Centro de Rehabilitación Psicosocial del Gironès-Pla de 1’Estany, IAS. Girona.

ROSER IZQUIERDO. Centro de Salud Mental Sant Martí Sud, IAPS. Barcelona.

EITHNE LEAHY. Institut Municipal d’Investigació Mèdica, IMIM. Barcelona.

CARME MASFERRER. Hospital de Día de Salud Mental, Centre Forum-IAPS. Barcelona.

PAZ FLORES. Hospital de Día de Salud Mental, Centre Forum-IAPS. Barcelona.

Correspondencia: Eduard Palomer Roca. Centro de Rehabilitación Psicosocial del Gironès-Pla de 1'Estany, IAS. Girona. C/Francesc Rogès, 29 Bajo. Tel. 972213155

Correo electrónico: 94914@imas.imim.es

** Recibido: 7/07/2009 